\title{
Inflammatory Pseudotumor of the Skull Base Involving Fissura Petrooccipitalis: A Rare Case with Challenging Diagnosis
}

\author{
Biao Huang, M.D., Ph.D., ${ }^{1}$ Hong-Jun Liu, M.D., Ph.D., ${ }^{1}$ \\ and Chang-Hong Liang, M.D., Ph.D.'
}

Inflammatory pseudotumor (IPT) is a benign entity that may present as a solid mass mimicking a malignant neoplasm. Histologically, they are composed of varying proportions of myofibroblastic spindle cells, lymphocytes, and plasma cells. Skull base IPT is rare and usually occurs in adults with no sex predilection. The skull base IPT typically presents with headache, and/or cranial nerve palsy. There is no consensus regarding treatment of skull base IPT due to its rarity. Surgical resection and corticosteroid therapy have generally been used. The preoperative diagnosis of skull base IPT is usually difficult due to its nonspecific clinical and radiologic features. We report a case of a 42-year-old woman with IPT originating from the skull base. To our knowledge, this is the first case of IPT invading fissura petrooccipitalis. When a mass in the skull base appears hypointense on T2-weighted imaging with aggressive growth and bony destruction, IPT should be considered in the differential diagnosis.

KEYWORDS: Inflammatory pseudotumor, skull base, magnetic resonance imaging, diagnosis

Inflammatory pseudotumor (IPT) is a benign entity that can mimic a malignant neoplasm. It has been described in both genders, at all ages, and in nearly every anatomic site. ${ }^{1}$ In the head and neck region, it is uncommon but most often affects the orbit. Skull base IPT is rare and usually pursues an aggressive course indistinguishable from malignant tumor. ${ }^{2}$ The lesion may involve adjacent tracts or cranial nerves. Most reports of skull base IPT have included only a single case or a small case series. Therefore, little is known concerning the natural history of this lesion. We present a rare case of IPT originating from the skull base, extending intracranially via the fissura petroocci- pitalis. To our knowledge, this is the first case of IPT invading the fissura petrooccipitalis. The radiographic presentations and clinical features are discussed. The literatures regarding pathologic mechanism, preoperative diagnosis, and treatment of skull base IPT are reviewed.

\section{CASE REPORT}

A 42-year-old woman with a history of left-sided headaches presented with new-onset left facial palsy and diplopia. For nearly 2 years before admission, the patient had complained of an idiopathic left occipital headache

\footnotetext{
${ }^{1}$ Department of Radiology, Guangdong Academy of Medical Sciences, Guangdong General Hospital, Guangzhou, Guangdong, China. Address for correspondence and reprint requests: Chang-Hong Liang, Department of Radiology, Guangdong Academy of Medical Sciences, Guangdong General Hospital, \#106 Zhongshan 2nd Road, Guangzhou, Guangdong, China 510080 (e-mail: dr_chhliang@ yahoo.com.cn).
}

Skull Base Rep 2011;1:105-110. Copyright (C) 2011 by Thieme Medical Publishers, Inc., 333 Seventh Avenue, New York, NY 10001, USA. Tel: $+1(212)$ 584-4662.

Received: December 14, 2010. Accepted after revision: February 10, 2011. Published online: July 7, 2011.

DOI: http://dx.doi.org/10.1055/s-0031-1280739.

ISSN 2157-6971. 
which could be relieved with Carbamazepine. She denied any history of associated fever, nausea, vomiting, or weight loss. A physical examination demonstrated left binocular horizontal diplopia as well as loss of sensation to her left face to light touch. Her cognitive function and tendon reflexes were normal. Epstein-Barr virus (EBV) capsid antigen $\mathrm{IgG}$ titers were 1:160, however, other laboratory investigations were within normal limits.

A CT scan revealed a soft-tissue density mass in the left skull base with enlargement of left fissura petrooccipitalis (Fig. 1). The lesion had also invaded the left clivus and left petrous apex. Magnetic resonance imaging (MRI) of her brain and nasopharynx was performed using a 1.5 Tesla scanner. MRI disclosed an irregular nodular mass in the same region. The mass measured $59 \mathrm{~mm} \times 26 \mathrm{~mm} \times 31 \mathrm{~mm}$ in size. It was hypointense compared with cerebral gray matter on T2-weighted images (Fig. 2A) and isointense on T1weighted images (Fig. 2B). Marrow replacement of the left clivus was noted on the T1-weighted images. The tumor exhibited moderately uniform enhancement (Fig. 2C). On postcontrast T1-weighted imaging, the tumor extended intracranially superiorly via an enlarged left fissura petrooccipitalis. The adjacent dura were thickened and enhanced (Fig. 2D). The bilateral cavernous sinuses were not involved by the lesion. The nasopharyngeal mucosa was not thickened.

The patient underwent partial resection of the lesion via the nasopharyngeal cavity. The lesion was firm with limited vascularity. On routine $\mathrm{HE}$-stained sections, the lesion was composed of spindle cells in a

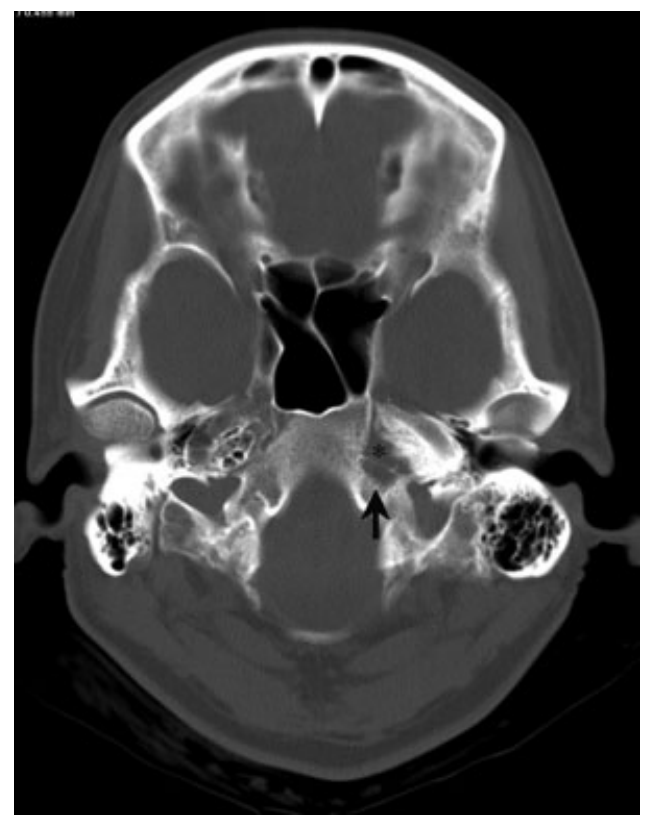

Figure 1 Noncontrast axial CT scan with bone windows reveals marked expansion of the left fissura petrooccipitalis (asterisk) and destruction of the left clivus (arrow) and left petrous apex. collagenous background of abundant lymphocytes, plasma cells with varying admixtures of collagen bundles (Fig. 3). Mitoses were very rare. The final pathologic diagnosis was inflammatory pseudotumor. The patient had no complications and was discharged on oral corticosteroids. Her symptoms of facial palsy and diplopia resolved completely 2 weeks after operation, and the mild residual pain disappeared after 1 month on corticosteroid treatment. The low-dose steroid maintenance therapy with prednisolone $10 \mathrm{mg}$ daily was continued for 10 weeks. Over 4 months after the operation, the patient was symptom-free and the follow-up MRI showed no sign of recurrence. Dural thickening and contrast enhancement was decreased.

\section{DISCUSSION}

IPT has been reported to occur most often in the lung, liver, orbit, mesentery, retroperitoneum, genitourinary tract, and upper respiratory tract. In the head and neck, IPT most commonly involves the orbit. Skull base involvement is rare. ${ }^{2,3}$ Skull base IPT usually occurs in adults with no sex predilection. Systemic symptoms such as fever or weight loss are uncommon in skull base IPT, but may occur in IPTs affecting other sites. The skull base IPT typically presents with headache, and/or cranial nerve palsy. ${ }^{4}$ Our patient presented with left-sided headaches and facial nerve palsies as the lesion was located in the left middle cranial fossa and had invaded the petrous bone and clivus. Cranial nerve palsies are most often due to the aggressive, invasive nature of skull base IPT.

The pathologic mechanism of IPT is still controversial. It is frequently associated with fever, sweats, fatigue, and lymphadenopathy, which suggests an infectious cause. Regardless of location, all IPTs share similar histological features. They are composed of varying proportions of myofibroblastic spindle cells, lymphocytes, and plasma cells. ${ }^{5,6}$ There is an absence of neoplastic cells. The isolation of EBV DNA from some cases of IPT suggests that EBV may play a role in at least a subset of IPT. ${ }^{7}$ The microorganisms are rarely detected in the IPTs, however. Acute immune and vascular causes of IPT have also been hypothesized. Our patient had an elevated EBV antigen IgG titer supporting a postinfectious cause. However, the term "inflammatory myofibroblastic tumor" has recently been suggested as a more descriptive name for inflammatory pseudotumor. Some authors believe this tumor is a low-grade fibrosarcoma with inflammatory cells. In 2002, the World Health Organization classified inflammatory myofibroblastic tumor as a soft-tissue tumor composed of differentiated myofibroblastic spindle cells, usually accompanied by numerous plasma cells and/or lymphocytes. ${ }^{8}$ Some studies have revealed cytogenetic clonal abnormalities and anaplastic lymphoma kinase expression suggesting a neoplastic etiology. 9,10 
A
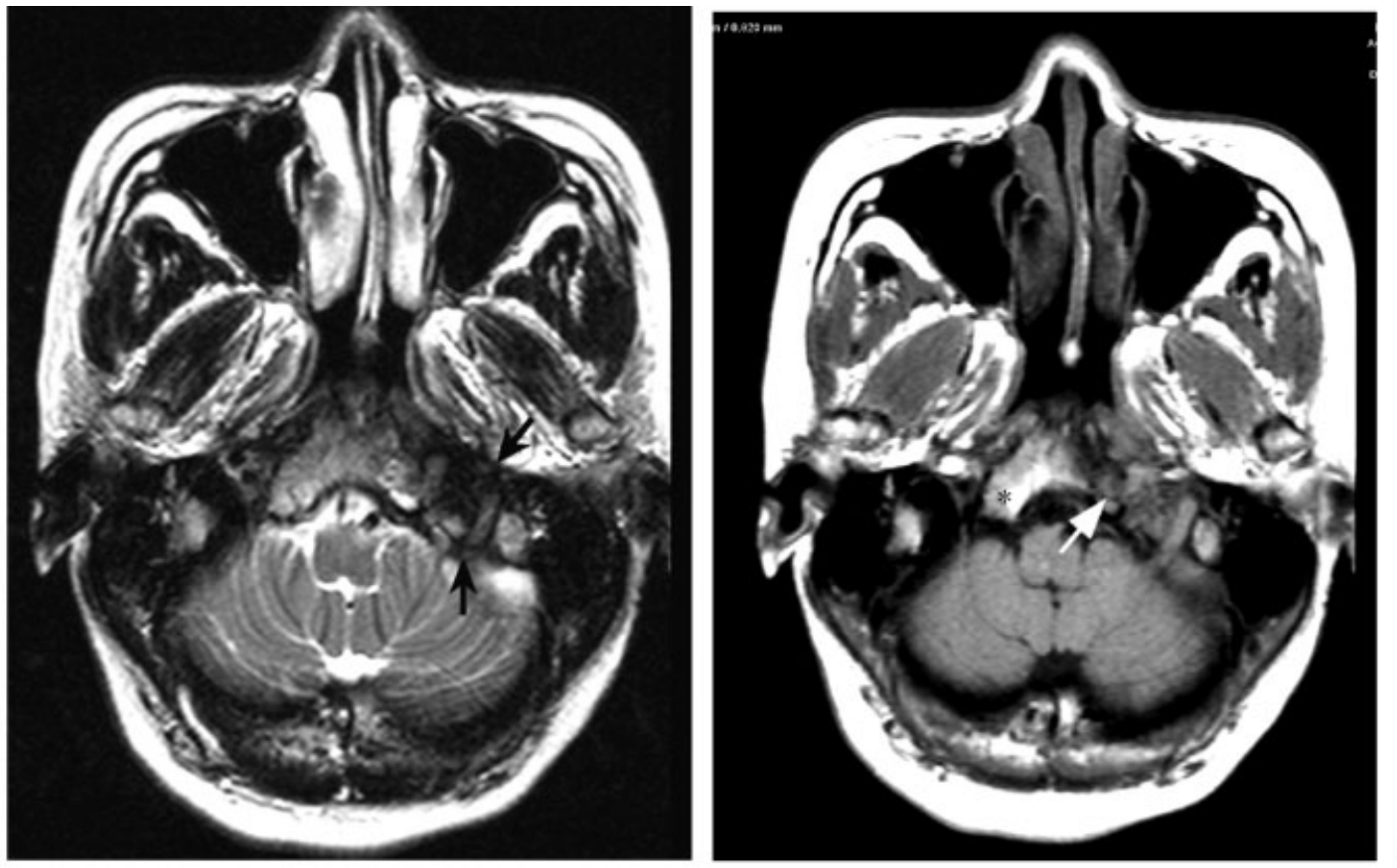

Figure 2 (A) Axial fast spin-echo T2-weighted MR image shows an expansile heterogeneous hypointense lesion (arrow) in the left fissura petrooccipitalis involving the left clivus. (B) Axial T1-weighted MR image as the same level as (A) shows the marrow replacement in the left clivus (arrow). The normal high signal intensity of bone marrow is seen on the right side (asterisk). (C) Axial postgadolinium T1-weighted MR image at the same level as (A) demonstrates moderately enhancement of the lesion (arrow). (D) Coronal postgadolinium T1-weighted MR image with fat suppression reveals the superior extension of the mass (arrow) with cerebral dural enhancement (arrowhead)

The preoperative diagnosis of skull base IPT is usually difficult due to its nonspecific clinical and radiologic features. Although they are histologically benign, IPTs often show aggressive clinical behavior, with locally destructive features that mimic a neoplastic process. In our case, for example, there were no signs or symptoms of infection (such as fever) and the tumor was originally thought to be a nasopharyngeal carcinoma (NPC). On CT scan, IPT frequently reveals a soft-tissue density mass with moderate enhancement. In contrast to orbital disease, skull base IPT presents with more aggressive growth and bony destruction, often accompanied by bony sclerosis. ${ }^{2,11}$ The bone sclerosis is suggestive of slow growth such as that seen in inflammatory conditions. MRI 


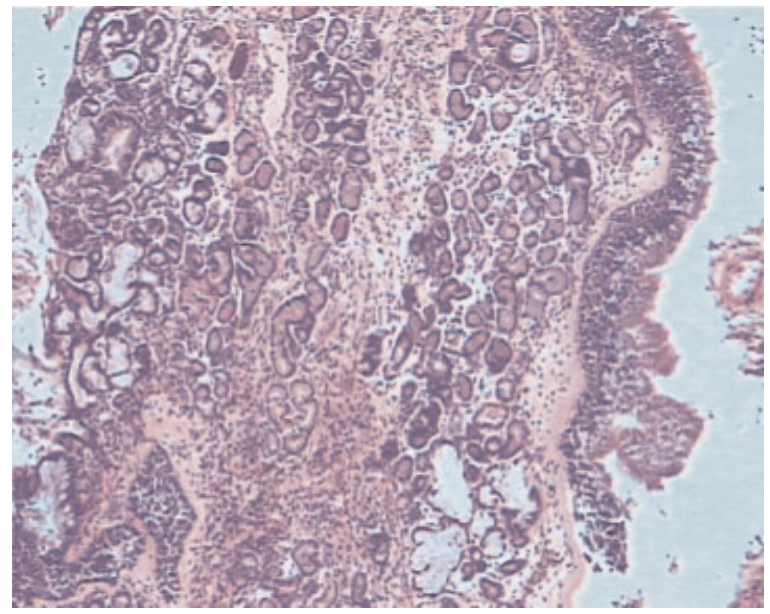

Figure 3 Photomicrograph of a histological section of the surgical specimen shows occasional spindle cells and admixed collagen bundles are present within a background of diffuse infiltration by lymphocytes and plasma cells (HE staining, original magnification $\times 200$ ).

features of skull base IPT are variable and are usually isoto hypointense relative to cerebral gray matter on both T1- and T2-weighted images. The presence of marked T2 hypointensity, as in our case, serves as an important clue in differentiating IPT from aggressive malignancies, such as infiltrative NPC, metastatic carcinoma, primary skull base lymphoma, or plasmacytoma, which are usually iso- to hyperintense on T2-weighted images. Lu et $\mathrm{al}^{12}$ reported seven patients with nasopharyngeal IPT and compared them with seven patients with NPC. All IPT lesions were hypointense to brainstem on T2-weighted images, and six of the NPC lesions showed mild hyperintensity on T2-weighted images. The remaining NPC lesion was isointense on $\mathrm{T} 2$-weighted images. Lee et a ${ }^{13}$ described a case of IPT originating from the clivus which also showed marked heterogeneous hypointensity on T2weighted imaging. The hypointensity seen on T2weighted images likely reflects a combination of fibrosis and attenuated cellularity. Intracranial extension with dural thickening and enhancement were observed in our case, which may be another feature of skull base IPT. Strasnick et $\mathrm{al}^{14}$ reported on two patients with temporal bone IPT and noted thickening and enhancement of adjacent intracranial dural structures in both cases.

There is no consensus regarding treatment of skull base IPT due to the rarity of the lesion. Complete surgical removal is usually attempted. If complete surgical resection of the lesion is impossible, administration of high-dose corticosteroid therapy is advocated. The response of IPT to corticosteroids is dependent on its histological features, as lesions with abundant lymphocytes and plasma cells are considered more sensitive to steroids compared with those that are predominantly fibrotic. ${ }^{15}$ In contrast to pseudotumors of the orbit, skull base IPT is less responsive to corticosteroid treatment.
Recurrences during the 2 years follow-up were as high as $15 \%$ in studies of extrapulmonary IPT. ${ }^{6}$ Long-term administration of low-dose prednisolone is recommended to prevent relapse. ${ }^{15}$ But the efficacy and safety of the low-dose steroid maintenance therapy in IPT still remains questionable. Radiation therapy has been used in patients with orbital IPT who cannot tolerate or fail corticosteroid treatment, ${ }^{14}$ but similar success has not been demonstrated in the limited number of reported cases of skull base IPT. In our case, the patient had significant clinical and radiologic improvement following surgical resection and responded well to subsequent corticosteroid therapy.

IPT rarely involves the skull base. When it does, it frequently presents with headache, and/or cranial nerve palsy due to its aggressive growth and bony destruction and can mimic a malignant tumor, radiologically. When a mass in the skull base appears hypointense on T2weighted imaging with associated thickening and enhancement of adjacent intracranial dural structures, IPT should be considered in the differential diagnosis. Complete surgical removal appears the first choice for therapy and subsequent steroid therapy may help to prevent recurrence.

\section{REFERENCES}

1. Narla LD, Newman B, Spottswood SS, Narla S, Kolli R. Inflammatory pseudotumor. Radiographics 2003;23(3): 719-729

2. Garg V, Temin N, Hildenbrand P, Silverman M, Catalano PJ. Inflammatory pseudotumor of the skull base. Otolaryngol Head Neck Surg 2010;142(1):129-131

3. McKinney AM, Short J, Lucato L, SantaCruz K, McKinney Z, Kim Y. Inflammatory myofibroblastic tumor of the orbit with associated enhancement of the meninges and multiple cranial nerves. AJNR Am J Neuroradiol 2006;27(10):2217-2220

4. Maruya S, Miura K, Tada Y, et al. Inflammatory pseudotumor of the parapharyngeal space: a case report. Auris Nasus Larynx 2010;37(3):397-400

5. Batsakis JG, el-Naggar AK, Luna MA, Goepfert H. "Inflammatory pseudotumor": what is it? How does it behave? Ann Otol Rhinol Laryngol 1995;104(4 Pt 1): 329-331

6. Coffin CM, Watterson J, Priest JR, Dehner LP. Extrapulmonary inflammatory myofibroblastic tumor (inflammatory pseudotumor). A clinicopathologic and immunohistochemical study of 84 cases. Am J Surg Pathol 1995;19(8):859-872

7. Arber DA, Weiss LM, Chang KL. Detection of Epstein-Barr Virus in inflammatory pseudotumor. Semin Diagn Pathol 1998;15(2):155-160

8. Häusler M, Schaade L, Ramaekers VT, Doenges M, Heimann G, Sellhaus B. Inflammatory pseudotumors of the central nervous system: report of 3 cases and a literature review. Hum Pathol 2003;34(3):253-262

9. Biselli R, Ferlini C, Fattorossi A, Boldrini R, Bosman C. Inflammatory myofibroblastic tumor (inflammatory pseudotumor): DNA flow cytometric analysis of nine pediatric cases. Cancer 1996;77(4):778-784 
10. Lawrence B, Perez-Atayde A, Hibbard MK, et al. TPM3ALK and TPM4-ALK oncogenes in inflammatory myofibroblastic tumors. Am J Pathol 2000;157(2):377-384

11. McCall T, Fassett DR, Lyons G, Couldwell WT. Inflammatory pseudotumor of the cavernous sinus and skull base. Neurosurg Rev 2006;29(3):194-200

12. Lu CH, Yang CY, Wang CP, Yang CC, Liu HM, Chen YF. Imaging of nasopharyngeal inflammatory pseudotumours: differential from nasopharyngeal carcinoma. Br J Radiol 2010;83(985):8-16
13. Lee JH, Kim K, Chung SW, Choi YC, Lee A. A case report of inflammatory pseudotumor involving the clivus: CT and MR findings. Korean J Radiol 2001;2(4):231-234

14. Strasnick B, Vaughan A. Inflammatory pseudotumor of the temporal bone: a case series. Skull Base 2008;18(1): 49-52

15. Lee DK, Cho YS, Hong SH, Chung WH, Ahn YC. Inflammatory pseudotumor involving the skull base: response to steroid and radiation therapy. Otolaryngol Head Neck Surg 2006;135(1):144-148 